\title{
1 EL ESTADO ARGENTINO Y EL DESARROLLO \\ UN ANÁLISIS DE LA CONFIGURACIÓN \\ E IMPLICACIÓN ESTATAL EN LA PROMOCIÓN INDUSTRIAL DURANTE LOS AÑOS 2003-2015
}

THE ARGENTIAN STATE AND DEVELOPTMENT. AN ANALYSIS OF THE STATE CONFIGURATION AND IMPLICATION FORMSFOR THE INDUSTRIAL PROMOTION DURING THE YEARS 2003-2015

\author{
María Jimena García Puente \\ Carolina Teresita Lauxmann \\ Emilia Ormaechea
}

\begin{abstract}
RESUMEN / En el marco de la recuperación de la importancia y la centralidad de los Estados para el desarrollo económico, que se manifestó en el escenario latinoamericano a principios del siglo XXI, el artículo analiza la configuración e implicación del Estado argentino en relación con una estrategia de desenvolviendo industrial que viabilice el desarrollo. Para ello, se examina cómo se organizó la estructura del Estado (configuración organizacional), las dotaciones presupuestarias (capacidad de fuego) de las dependencias/oficinas destinadas a la promoción industrial y las características de las políticas públicas industriales (formas implicativas). A partir de dicho análisis, se argumenta que las particularidades que asumieron la organización e implicación del Estado argentino entre 2003 y 2015 no habilitaron la conformación de una estrategia de acumulación basada en el desarrollo del sector industrial que permita la salida de posicionamientos periféricos.
\end{abstract}

PALABRAS CLAVE / estado; desarrollo productivo; Argentina

\begin{abstract}
In the framework of the recovery of the importance of the states for economic development, experienced in Latin American at the beginning of the XXI century, the paper analyzes the Argentinian state's configuration and its modalities of implication in order to promote industrialization as a strategy for development. To do so, the configuration of the state's structure (its organizational configuration), the budget of the offices and dependencies related to industrial promotion (fire capacity), and the characteristics of industrial policies (implicative forms) are analyzed. From this analysis, it is argued that the particularities of the organizational and implicative forms assumed by the Argentinian state during 2003-2015 did not allow the deployment of an accumulation strategy based on the development of the industrial sector that enables an overcome of the peripheral positioning.
\end{abstract}

KEY WORDS / state; productive development; Argentina 


\section{Introducción}

A principios del siglo XXI, el debate en torno a la importancia y centralidad de los Estados para la promoción del desarrollo económico volvió a ocupar un lugar relevante en la agenda política y académica latinoamericana, luego de quedar desplazado por más de casi tres décadas de primacía de políticas neoliberales en la región.

En términos políticos, dicho reposicionamiento estuvo asociado a las victorias electorales de diversas «reacciones» neodesarrollistas ${ }^{1}$ (BresserPereira, 2006, 2007; Aránibar y Rodríguez, 2013; Gaitán 2013) que, en el plano del discurso, cuestionaron fuertemente las políticas neoliberales de los años 90 y se posicionaron a favor de la recuperación de la intervención activa del Estado - vis á vis el mercado- para la promoción del crecimiento económico y el desarrollo de los países de América Latina.

Por su parte, en el ámbito académico, nuevamente adquirieron relevancia las discusiones en torno a la centralidad del Estado y la actividad industrial para el desarrollo, recuperándose los debates de las corrientes desarrollistas y estructuralistas latinoamericanas que tuvieron su apogeo durante las décadas de 1950-60 (Bresser-Pereira, 2007; Cao, Rey y Laguado Luca; 2015; Español y Herrera, 2011; Ferrer 2010; Nahón, Rodríguez y Schorr, 2006).

Argentina no se mostró ajena a este escenario. El período que inicia con el triunfo de Nestor Kirchner en 2003, y que continúa con las presidencias de Cristina Fernández de Kirchner hasta finales de 2015, se caracterizó por una recuperación, al menos en términos discursivos, de la centralidad del Estado para dinamizar proceso de desarrollo sobre la base del impulso y el direccionamiento del sector industrial (Ministerio de Industria, 2011; Kulfas, 2016).

En un primer momento, esta estrategia mostró buenos resultados en términos de crecimiento económico y reducción de la pobreza y la desigualdad (Kulfas, 2016). Pero, ya a inicios de la segunda década de la centuria, emergieron diversos problemas, muchos de los cuales estaban vinculados con la imposibilidad de avanzar en la conformación de una estrategia acumulativa sobre la base del desarrollo del sector manufacturero, que permita

1 En referencia a los gobiernos de: Hugo Chávez en Venezuela asumido en el año 1999; Ricardo Lagos en Chile en el 2002 (seguido por Michelle Bachelet en 2006); Luizl nácio Lula Da Silva en Brasil, en 2003 (seguido por Dilma Rousseff en el 2011); Nestor Kirchner en Argentina en 2003 (seguido por Cristina Fernández en 2007); Evo Morales en Bolivia en 2003; Tabaré Vázquez en Uruguay en 2004, Rafael Correa en Ecuador en 2007. 
dar sostenibilidad a los procesos de crecimiento con equidad distributiva (Kulfas, 2016; Porta, Santarcángelo y Schteingart, 2016).

A los fines de comprender esas dificultades, el artículo presenta un análisis de las particulares formas organizativas y lógicas implicativas desplegadas por el Estado argentino durante los años 2003-2015, en tanto se entiende que las mismas resultan centrales para (in)viabilizar determinadas estrategias de acumulación. En ese sentido, se argumenta que las características que asumieron la organización e implicación estatal entre 2003 y 2015 no habilitaron la conformación de una estrategia de acumulación basada en el desenvolvimiento del sector industrial que viabilice el desarrollo.

Para sustentar dicha argumentación, el trabajo se estructura de la siguiente manera. Un primer apartado en donde se recuperan las perspectivas que examinan al Estado como un actor central en la configuración procesos de desarrollo y dan cuenta de la importancia de la conformación organizativa y las formas implicativas estatales para habilitar u obturar diferentes estrategias de acumulación. Asimismo, se avanza en la consideración de la relevancia del fomento al sector industrial como un aspecto central de las estrategias de desarrollo. Un segundo apartado, en el cual se analiza el rol que asumió el Estado argentino en la promoción del desarrollo industrial durante el periodo denominado neodesarrollista. Para ello, se examina cómo se organizó la estructura del Estado (configuración organizacional), las dotaciones presupuestarias (capacidad de fuego) de las dependencias/oficinas destinadas al fomento de la actividad económica y las características de las políticas públicas industriales (formas implicativas). Un tercer apartado en el que se examina la estrategia de acumulación resultante de la particular forma organizativa y lógica implicativa del Estado durante el interregno 2003-2015. Por último, se presentan unas breves consideraciones finales.

\section{La organización e implicación estatal y la industrialización}

El papel del Estado para incidir en los procesos de desarrollo social y económico fue examinado desde diversas vertientes teóricas provenientes de la sociología histórica, la economía política y la ciencia política. Esos abordajes, con sus variantes, analizan al Estado en tanto un conjunto diferenciado de estructuras, recursos y poder, capaz de llevar adelante políticas públicas que configuran a la sociedad (Skocpol, 1991; Mann, 1991), generan transformaciones estructurales y promueven el desarrollo económico (Evans, 1995, 1996). 
En diálogo con estas perspectivas, emergieron trabajos que examinaron los casos exitosos del este asiático, en donde se desataca el papel del Estado y de sus cualidades organizativas y de sus vínculos con los actores económicos en el direccionamiento de la estrategia de desarrollo. En esos escenarios, la intervención cualificada del Estado dio lugar a un cambio estructural, a partir del desenvolvimiento del sector industrial centrado en bienes de alto contenido tecnológico y valor agregado, orientado a mejoramiento de la competitividad internacional (Amsden, 1989; Chan, 2004; Evans, 1995; 1996; Wade, 1999).

Las características de los Estados, en términos de estructuras organizativas y de dotaciones presupuestarias, son las que - de acuerdo con los enfoques señalados ${ }^{2}$ - inciden en la formulación e implementación de las políticas públicas. De acuerdo con ello, una estructura que presente una nodalidad estratégica (Chibber, 2004) y cuente con recursos financieros necesarios para alcanzar los objetivos (Skocpol, 1991) posee la potencialidad de lograr una implicación coherente y cohesionada que habilite una estrategia de acumulación que viabilice el desarrollo (Kolhi, 2010).

De este modo, se evidencia que una forma de organización (configuración estatal) y un tipo de intervención socioeconómica (modalidad implicativa) $)^{3}$ se vinculan con la potencialidad de habilitar diferentes estrategias de acumulación, es decir, una particular forma de hacer funcionar la acumulación capitalista, garantizando la continua valorización del capital productivo - o industrial- como motor del proceso de acumulación.

Ahora bien, en los debates recientes sobre el desarrollo en América Latina se encuentran argumentaciones y propuestas que recuperan las experiencias el Este Asiático (Fernández y Lauxmann, 2014; Sevares, 2010), junto con las contribuciones del estructuralismo latinoamericano (Bresser-Pereira, 2007; Gaitán, 2014), que refuerzan la importancia de la industrialización para viabilizar la salida de posicionamientos periféricos.

De acuerdo con el estructuralismo latinoamericano, para alcanzar el desarrollo se requería que las estructuras productivas logren mayores nive-

2 Gran parte de esos argumentos dieron base a una vasta cantidad de estudios sobre capacidades estatales de diversos sectores de políticas. Específicamente, destacamos los vinculados con el desarrollo industrial en América Latina (Fernández, et al 2006, Sikkink, 1993, Castellani y LLampart, 2012, AvilaGomite y Boschi (ed.) 2016).

3 Dados los límites de este trabajo, nos centramos en los aspectos organizacionales e implicativos en relación con la posibilidad de habilitar estrategias de desarrollo, y no en el análisis de que dicha configuración estatal es resultado de un proceso político, siempre abierto y disputado, en el que se enfrentan diferentes clases, fracciones, grupos y movimientos sociales de base nacional e internacional. 
les de homogeneidad, diversificación y capacidad de generación de empleo de elevada productividad. Esto demandaba, centralmente, avanzar en un proceso de industrialización que permita romper con las formas de articulación a la economía internacional vigente por entonces (CEPAL, 1951; Prebisch, 1949). Al promover el desarrollo del sector manufacturero se abordaba, directamente, el problema de la especialización de la estructura productiva interna y la heterogeneidad estructural (Pinto, 1973). En ese sentido, la propuesta era elevar la cantidad de trabajadores desempeñados en actividades con mayores niveles de productividad (en el sector industrial), a los fines de poder incrementar el producto total y mejorar el nivel de vida de gran parte de la población, para lo cual esta corriente apelaba a un activo papel del Estado. El Estado tenía un rol fundamental en la programación de dicha estrategia de desarrollo, a partir de establecer las metas y los objetivos, y coordinar la ejecución de las acciones tendientes a alcanzarlos (CEAPL. 1955; Prebisch, 1952).

El sector manufacturero continúa teniendo una relevancia central en la problematización del desarrollo latinoamericano en la economía capitalista contemporánea. Entre las diversas razones que permiten afirmar que aún, hoy día, las «manufacturas importan» (Cohen y Zysman, 1987) puede señalarse, tal como lo indicó oportunamente Raúl Prebisch (1949), que el sector manufacturero continúa teniendo un peso central en la generación de empleo de elevada productividad, ya sea de manera directa, o bien a través del estímulo al desarrollo del sector servicios (Helper, Krueger y Wial, 2012). En ese sentido, el desarrollo del sector manufacturero actúa como estimulante de la demanda de servicios cada vez más sofisticados, como pueden ser, los de ingeniería, diseño, e investigación y desarrollo (Amsden, 2004; Fernández, 2017; Hikino y Amsden, 1995; Lauxmann y Fernández, 2015). A su vez, estas áreas promueven la generación continua de innovaciones y de progreso técnico, permitiendo mantener al conjunto de la estructura productiva en la frontera tecnológica (Lavarello, 2017). Por su parte, también es importante destacar el rol del sector como correa de transmisión entre las oportunidades científicas y técnicas y el resto del sistema productivo. Es decir, la actividad manufacturera es la que tiene la capacidad de producir insumos, como máquinas o productos químicos o biológicos, que explican la aplicación de técnicas y los aumentos de productividad en otros sectores (Greenwald y Stiglitz, 2006).

Pero, como ha evidenciado la experiencia histórica latinoamericana, y advierte una vasta literatura (ver por ejemplo Amsden, 2004; Arceo, 2005; Arrighi, Silver y Brewer, 2003; Fajnzylber, 1983,1992; Hausmann y Rodrick, 2003), no cualquier tipo de actividad manufacturera impulsa la salida de 
posicionamientos periféricos. Particularmente importante, para viabilizar el desarrollo de los países de la región, resulta el impulso de una actividad manufacturera endógena, compleja y dinámica. Endógena, porque se trata de un proceso de desenvolvimiento del sector industrial en el que prima el capital doméstico; compleja, porque genera la capacidad de establecer eslabonamientos intra e intersectoriales; y dinámica, porque para su reproducción lleva adelante prácticas vinculadas al desarrollo de innovaciones y nuevas tecnologías (Fernández, 2017).

En consecuencia, luego de haber planteado estas consideraciones, la pregunta que surge es, en el marco de la recuperación y re-apelación política y académica del papel del Estado en la generación del desarrollo sustentado en el sector industrial ¿Cómo se organizó la estructura del Estado para llevar adelante dicha estratégica? ¿Cuáles fueron los cambios de jerarquías organizacionales? ¿Con qué dotación presupuestaria contaron dichas dependencias? ¿Cuáles fueron las políticas industriales, la modalidad de implicación que llevaron adelante esas estructuras? Una vez que se avance en ello, se examina cuál fue la estrategia de acumulación que habilitó dicha modalidad organizativa e implicativa del Estado argentino. Tal estrategia ¿modificó las estructuras productivas de la economía argentina?

\section{El Estado y las estrategias de acumulación en Argentina a inicios del siglo XXI}

La estructura organizacional del Estado y su capacidad de fuego

A lo largo de la primera década del siglo XXI, el aparato estatal argentino se ha ido robusteciendo en términos de estructura organizativa. Ejemplo de ello ha sido el incremento en volumen e importancia de la estructura ministerial, en procura de la jerarquización de determinadas funciones a realizar por el aparato estatal, a través de la creación de ministerios, secretarías y subsecretarías.

En esta expansión del aparato estatal se puede advertir su intención de implicarse en el fomento de la actividad productiva-industrial para, a partir de ello, estimular el desarrollo. En el año 2002 se crea el Ministerio de la Producción, con oficinas vinculadas específicamente al impulso de la industria. Luego, durante los años 2003-2008, esa cartera ministerial y las dependencias vinculadas a industria pasaron a estar bajo la órbita del Ministerio de Economía y Producción. En 2008 se crea nuevamente el Ministerio de Producción, el que asume, una vez más, las instancias vinculadas a la acti- 


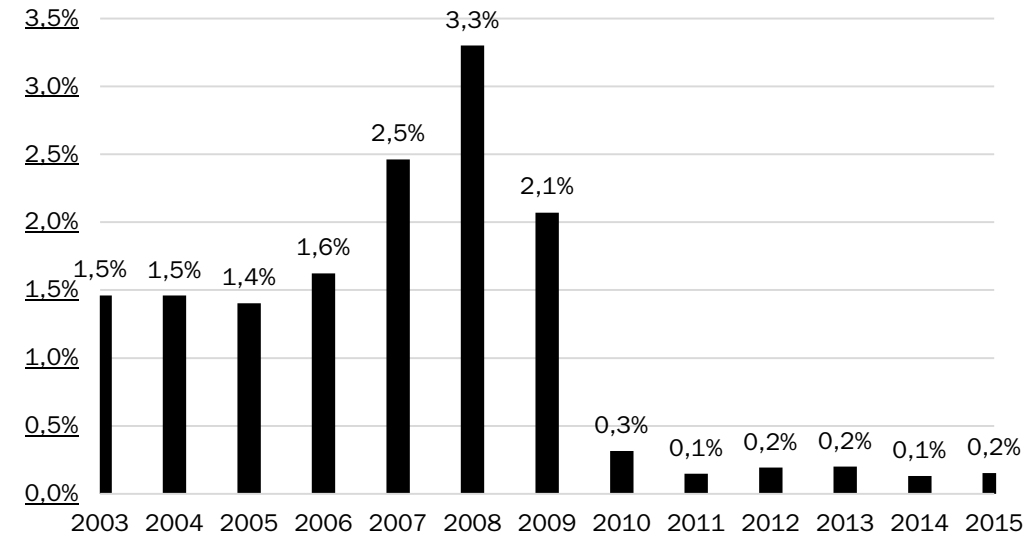

Gráfico 1. Evolución del Gasto Público de los ministerios que tuvieron bajo su órbita de influencia oficinas vinculas al desarrollo industrial durante el período 2003-2015, como porcentaje del gasto total.

Fuente: Elaboración propia en base a datos de la Oficina Nacional de Presupuesto del Ministerio de Economía de la Nación.

vidad manufacturera. Finalmente, en 2009, tiene lugar la instauración del Ministerio de Industria, el que durante un breve interregno, ese mismo año, opera bajo la denominación de Industria y Turismo, para finalmente entre 2010 y 2015 consolidarse como Ministerio de Industria.

Esas modificaciones y rejerarquizaciones de las áreas vinculadas con el desenvolvimiento del sector industrial, a pesar que dieron un espacio específico de intervención, no le otorgaron un lugar central en el conjunto de la estructura del Estado nacional. Puede advertirse que el Ministerio (con sus secretarías y subsecretarías) vinculado a la actividad productiva industrial, representó sobre finales de la gestión de Cristina Fernández de Kirchner el 4,5\% del total de la estructura estatal.

Por otro lado, se advierte la escasa capacidad de fuego de los ministerios que, durante los años 2003-2015, tuvieron bajo su órbita de influencia las oficinas vinculadas a la actividad industrial. Es decir, los recursos financieros con que contaron estas instancias para hacer frente a las actividades tendientes a cumplir con sus objetivos, evidenciaron un porcentaje muy bajo del total de gastos presupuestarios. Esta escasa gravitación puede advertirse particularmente luego de 2009, cuando se crea el Minis- 


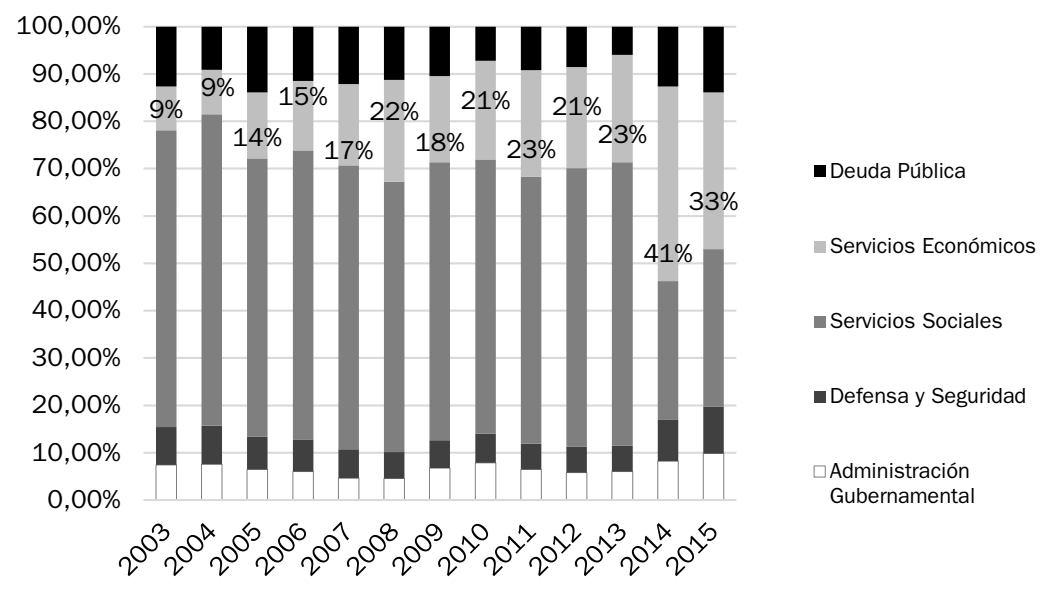

Gráfico N². Gasto Público Nacional por finalidades, porcentajes de participación 2003-2015.

Fuente: Elaboración propia en base a datos de la Oficina Nacional de Presupuesto del Ministerio de Economía de la Nación.

terio de Industria como cartera diferenciada del Ministerio de la Producción, tal como resulta del Gráfico $\mathrm{N}^{\circ} 1$.

Por otra parte, cuando se analiza más allá de las instancias ministeriales que directamente están implicadas en la intervención en la esfera industrial, y se realiza un examen del gasto por finalidades, se advierte un importante crecimiento de la participación de servicios económicos dentro del total del gasto público nacional (ver Gráfico №²). En el año 2003, el gasto en servicios económicos representaba un 9,30 \% del total del presupuesto nacional, en el año 2014 dicho porcentaje ascendió a 41,09 \% y en 2015 fue de $33,15 \%$.

Sin embargo, al profundizar el análisis al interior de servicios económicos, se observa que los flujos destinados específicamente a la actividad industrial revisten poca importancia, representando, en promedio, para el período 2003-2015 sólo el 1,8 \% del total de dicha finalidad. El grueso del gasto público de servicios económicos durante esos años estuvo vinculado a las funciones de Energía, Combustible y Minería y Transporte, que representó el 79,3\% del total del presupuesto (Gráfico № 3 ). Ello se encuentra asociado a la existencia de importantes subsidios al consumo de electricidad y/o de transporte vigentes durante el interregno antes mencionado. 


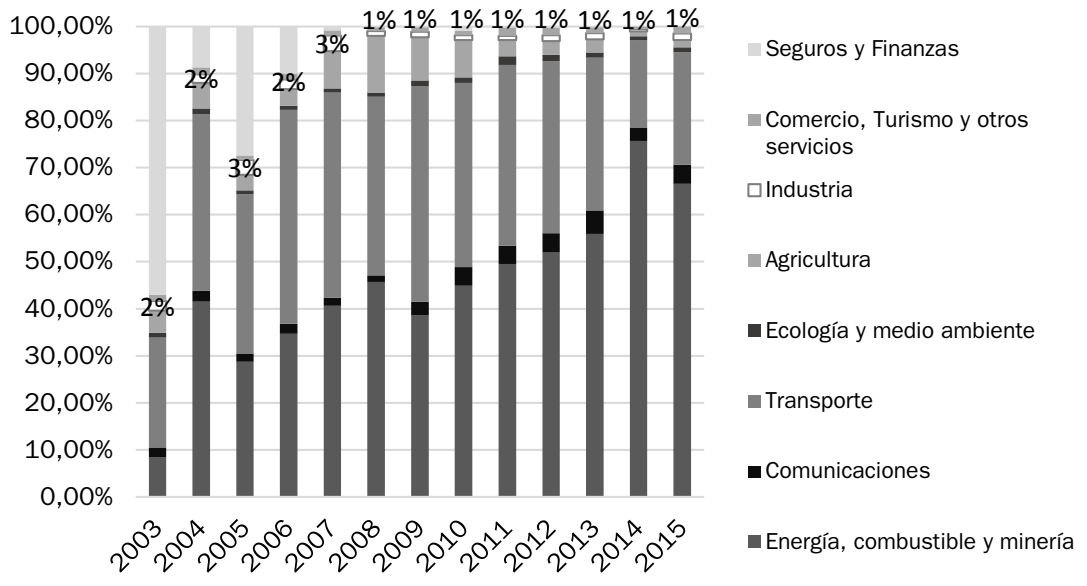

Gráfico N³. Gasto Público Nacional en Servicios económicos desagregado por funciones, 2003-2015.

Fuente: Elaboración propia en base a datos de la Oficina Nacional de Presupuesto del Ministerio de Economía de la Nación.

Se observa, así, que se han jerarquizado en el organigrama las dependencias destinadas a incidir en la actividad productiva-industrial, asumiendo especificidad y rango ministerial. No obstante ello, tales oficinas no adquirieron centralidad dentro de la estructura del Estado. Además, el examen de la capacidad de fuego de estas instancias ministeriales tampoco muestra una tendencia a su empoderamiento dado los bajos porcentajes que evidencian conforme a la clasificación institucional del gasto público. Resta resaltar que cuando se analiza el presupuesto por finalidad, si bien se advierte una creciente participación de servicios económicos dentro del total, al examinar las funciones específicas del gasto público, se observa la baja gravitación que presenta el estímulo al sector industrial.

Las modalidades implicación del Estado argentino.

Las políticas industriales

En referencia a las formas de implicación del Estado argentino a inicios del siglo XXI, en lo relativo al fomento de la actividad industrial, cabe destacar que la principal acción para impulsar el despegue y el desarrollo de la industria fue el mantenimiento de un dólar competitivo. En ese sentido, 
esa política macroeconómica posibilitó la expansión y crecimiento del sector industrial durante la fase inicial del período.

Luego, a partir del año $2008^{4}$ se desarrollaron distintas acciones estatales tales como la restricción a las importaciones, aumentos de aranceles, direccionamiento de inversiones productivas junto con políticas específicas del fomento al sector industrial. No obstante ello, diversos autores (Azpiazu y Schorr, 2010; Baruj, Kosacoff y Ramos, 2009; Couto, 2010; Fernández Bugna y Porta, 2007; Kulfas, 2009; Rougier y Schorr, 2012) marcan la ausencia de un abordaje estratégico, integrado y global hacia el sector industrial, y señalan la presencia de un conjunto fragmentado y descoordinado de políticas y programas específicos que, con dispar éxito, se desarrollaron en esta fase.

La mayoría de ese conjunto de políticas no fueron nuevas, han sido formuladas durante el periodo neoliberal para resolver «fallas» del mercado y limitaciones de algunas empresas; tenían un alcance horizontal (sus destinatarios eran las empresas independientemente de su inserción sectorial o actividad), sin definiciones de sectores prioritarios (Couto, 2010; Kulfas, 2009; Lavarello y Goldstein, 2011). Pero cabe indicar que, en el periodo bajo análisis, tales programas incrementaron sus recursos financieros/económicos y aumentaron sus destinatarios (García Puente, 2016).

Junto con ello, también se llevaron adelante acciones sectoriales y de fomento de competencias tecnológicas y modernización, aunque ocupando un lugar menor que las horizontales. Las mismas se orientaron: i) a promover sectores vinculados con la investigación, el desarrollo y la exportación - el caso de software, la biotecnología-, ii) a actividades con potencial crecimiento de la demanda o del empleo - el mantenimiento del régimen de privilegio para el sector automotor y motopartes-, iii) a segmentos con ventajas naturales de gran escala - como la minería metálica o el sector forestal-, y iv) a atender situaciones de crisis - como maquinaria agrícola o tabaco- (Baruj y Porta, 2006; Couto, 2010). Los regímenes de promoción sectoriales se presentaron a través de incentivos fiscales y arancelarios. Asimismo se implementaron regímenes de promoción industrial ${ }^{5} \mathrm{y}$ se mantuvieron los regímenes de promoción regional para las radicacio-

4 Cuando se agotaron los beneficios del dólar alto y se presentó una crisis económica a nivel mundial que tuvo repercusiones en Argentina (Castells y Schorr, 2015).

5 Régimen de Incentivos para los fabricantes nacionales de bienes de capital (decreto 379/01). Luego, con la Ley de Promoción Industrial (Ley 25.924 del año 2004), en el año 2008, se continuaron con la Ley de Promoción de Inversiones en Bienes de Capital y Obras de Infraestructura (Ley $\mathrm{N}^{\circ}$ 26.360). 
nes industriales en las provincias de La Rioja, Catamarca, San Juan y San Luis, se extendió el régimen ensamblador de Tierra del Fuego y el estímulo a las exportaciones por puertos patagónicos.

En referencia al sector Pymes, con la sanción de una nueva Ley de Pymes 25300 en 2000 , se crearon nuevas políticas y se modificaron algunas existentes, que recién a partir del año 2006 se comenzaron a implementar y a ejecutar presupuestariamente. No obstante ello, las políticas públicas hacia Pymes tuvieron un limitado alcance, no se encontraban articuladas conceptualmente con el resto de las intervenciones industriales y de desarrollo productivo, y operaron fragmentadamente (Kulfas, 2006).

Por otro lado, se identifican esfuerzos por extender las políticas de acceso al financiamiento para la inversión y del desarrollo. Entre las que se destacan el Programa del Financiamiento Productivo del Bicentenario (Decreto 783/2010); la reforma de la carta orgánica del Banco Central de la República Argentina en marzo de 2012, Ley 26 739, y el FONDEAR (Fondo para el Desarrollo Económico Argentino).

Las intervenciones estatales llevadas adelantes desde el Ministerio de Industria en este período se centran en los siguientes ejes (ver cuadro 1): el desarrollo de acciones vinculadas con la sustitución de importaciones,

- FONAPYME Eficiencia Energética

- FONAPYME Industria

- Fondo Nacional para el Desarrollo y Fortalecimiento de las MiPyMEs (FONDyF)

- Programa de Financiamiento para la Ampliación y Renovación de Flota

- Registro Nacional de Laboratorios de Ensayos de Autopartes y Vehículos completo

- INCUBAR

- Mi PC Móvil

- Régimen de Crédito Fiscal para Capacitación

- Programa Nacional de Capacitación

- Régimen de Consolidación de la Producción Nacional de Motores y Cajas de Transmisión

- Régimen de Fortalecimiento del Sector Autopartista Argentino

- Proyecto Desarrollo Industrial Sostenible (DIS)

- Régimen de Crédito Fiscal para Instituciones

- FONAPYME Fondo Nacional para el Desarrollo de la Micro, Pequeña y Mediana Empresa

- FOGAPYME Fondo de Garantía para la Micro, Pequeña y Mediana Empresa

Programa Mi Galpón

- RBT Régimen de Bonificación de Tasas

- Programa Nacional para el Desarrollo de Parques Industriales Públicos en el Bicentenario. RENPI

- Programa Expertos PYME 
- PACC- Empresas. Programa de Acceso al Crédito y la Competitividad - Empresas: Acceso a las MiPyMES

- Red de Agencias de Desarrollo Productivo

- Programa Nacional para el Desarrollo de Parques Industriales Públicos en el Bicentenario - PyMEs

- Programa Nacional para el Desarrollo de Parques Industriales Públicos en el Bicentenario - ANR para Obras Intramuros: \$

- Aprendiendo a Emprender

- SGR. Sociedades de Garantía Recíproca

- Régimen Compre Trabajo Argentino

- Auspicios de la Secretaría de Industria

- Autorización de Importación de Autopartes de Seguridad que No Requieren CHAS

- Desarrollo de Proveedores Industriales Nacionales

- Capital Semilla

- Empresas Madrinas

- Programa de Cooperación Industrial Internacional - PCII

- PACC- Emprendedores. Programa de Acceso al Crédito y la Competitividad Emprendedores

- Plan Nacional de Diseño

- Ley de Promoción de la Industria del Software

- Exención de Gravámenes

- Eximición de Derechos de Importación para Radioaficionados

- Grupo de PyMEs y Cooperativas: Sistemas Productivos Locales

- Importación de Productos Destinados a la Rehabilitación, al Tratamiento y la Capacitación de Personas con Discapacidad

- Importación de Vehículos para Exhibición

- Programa Mi Pc

- Régimen de Incentivo a la Producción de Bienes de Capital, Informática y Telecomunicaciones

- Proyecto DIS - Programa de Apoyo para el Desarrollo Industrial Regional

- Proyecto DIS - Programa Innovación en Cadenas de Valor

- Régimen Aduana en Factoría

- Régimen de Importación de Vehículos de Colección

- RIN Registro Industrial de la Nación

- Régimen Saldo Técnico IVA

- Régimen para Vehículos Especiales Usados

- Registro de Importación del Sector Editorial

- Registro de Armas Químicas

- Licencias de Configuración de Modelos y Constancias Técnicas

- Importación Temporal

- Régimen de la Industria Naval

- Importación de Bienes de Capital Usados Reacondicionados

- Exportación Temporal

- Certificado de Homologación de Autopartes de Seguridad

- Unidad de Medio Ambiente

Cuadro N 1. Políticas públicas nacionales del Ministerio de Industria. Año 2014. Fuente: www.industria.gov.ar 
el fomento a los parques industriales, el diseño del Plan Estratégico 2020 y el desarrollo federal.

De acuerdo con lo indicado, se puede considerar que la modalidad de implicación estatal da cuenta de una importante presencia del Estado en la promoción del sector productivo industrial. Sin embargo, ello no generó una forma de implicación coherente que se oriente hacia la construcción de una estrategia de acumulación centrada en el sector manufacturero, sino que se caracterizó por presentar la forma de un conjunto de acciones que operaron fragmentada y descoordinadamente.

\section{La estrategia de acumulación resultante}

La configuración organizacional, la capacidad de fuego y las modalidades de implicación del Estado argentino en los años 2003-2015 no han permitido conformar una estrategia de acumulación que, sobre la base del desarrollo del sector manufacturero, permita viabilizar el desarrollo. Sostenemos ello a pesar de las importantes tasas de crecimiento del PBI durante el periodo señalado, motorizadas, en gran medida, por la recuperación de la actividad industrial. ${ }^{6}$

Como se puede advertir en el Gráfico $\mathrm{N}^{\circ}$ 4, tomando la serie del Banco Mundial UMN a precios constantes, el crecimiento del sector manufacturero fue un elemento central en el crecimiento del período. Promediando tasas de crecimiento del 7,93 \% entre $2003-2008$ el sector industrial recuperó participación dentro del PBI, el cual creció a un promedio del 7,80\% en dicho período. Tras una importante caída en 2009 del 7,26 \% para el Producto Industrial y del 5,92 \% para el PBI global, los mismos se recuperaron brevemente en 2010 y 2011 para luego volver a caer 2012 y 2014 y ralentizar su marcha hacia finales de 2015.

El crecimiento del sector manufacturero en el período poscrisis del neoliberalismo ha estado sustentando en un incremento de la producción del conjunto de las actividades manufactureras (Azpiazu ySchorr, 2010). No obstante, también puede advertirse que hay ciertas ramas industriales que han experimentado un crecimiento más acelerado que otras (al menos en los primeros años poscrisis 2001). Las actividades manufactureras que habían resultado fuertemente castigadas por la reestructuración neoliberal (textil

6 En el crecimiento de este período también ocupó un lugar central el precio de los commodities, principalmente de la soja. El precio de la misma en el año 2002 era de aproximadamente 200 USD por tonelada, en el 2008 alcanzó los 622 USD, y en el 2015 bajó a 340 UDS. 


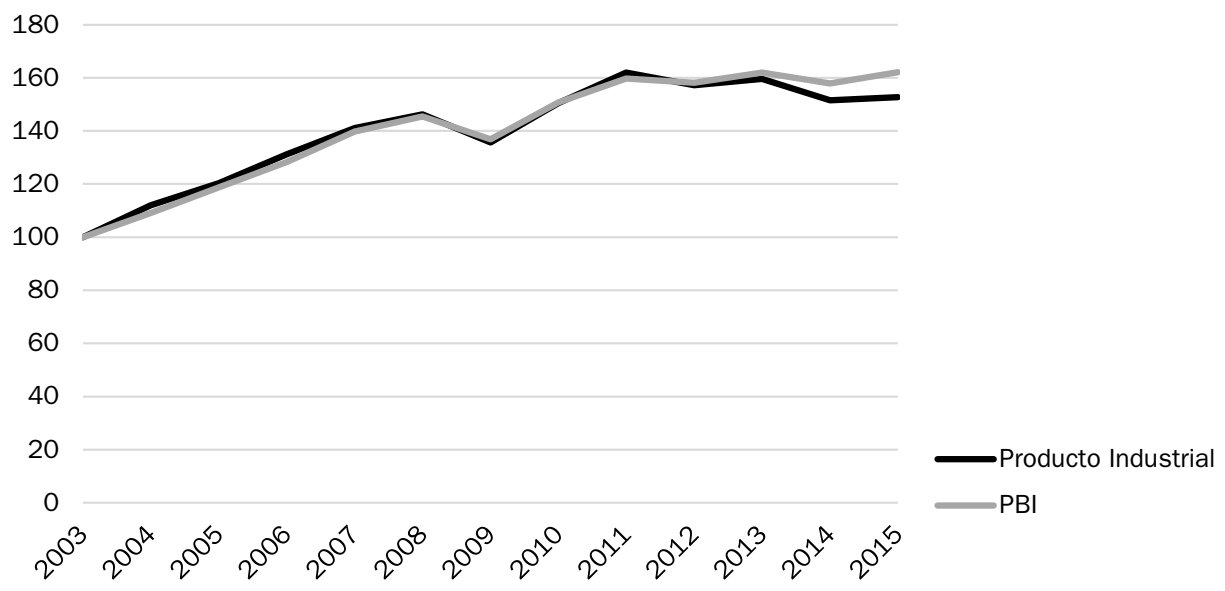

Gráfico N4. Evolución de la Producción Manufacturera y el PBI, 2003-2015 (índice base $2003=100$ ).

Fuente: Elaboración propia sobre la base de información del Banco Mundial.

e indumentaria, productos metálicos, máquinas y equipos, por mencionar algunas de las más importantes) han contribuido significativamente al crecimiento del sector en los inicios de la actual fase de reactivación industrial (Azpiazu ySchorr, 2010; Fernández Bugna y Porta, 2007). El crecimiento de estas ramas respondió básicamente a la utilización de la capacidad ociosa instalada, aunque también incidió en su desenvolvimiento la apertura de nuevas empresas (Kulfas, 2009; Schorr, 2012).

Sin embargo, tal crecimiento no modificó la composición intrasectorial de las manufacturas. Se puede advertir (ver Cuadro $\mathrm{N}^{\circ} 2$ ) la persistencia de la especialización del sector en actividades que sustentan su competitividad en ventajas comparativas naturales, y/o amparadas por un proteccionismo frívolo que crea "ventajas institucionales de privilegio» (Azpiazu y Schorr, 2010; Rougier y Schorr, 2012).

Por otra parte, estas actividades industriales presentan un elevado grado de concentración en un reducido grupo de actores: grandes empresas, crecientemente de capital extranjero (ver Cuadros $\mathrm{N}^{\circ} 3$ y $\mathrm{N}^{\circ} 4$ ), que detentan posicionamientos monopólicos u oligopólicos en los mercados locales (por lo que obtienen rentas extraordinarias) (Azpiazu y Schorr, 2010; Rougier y Schorr, 2012), lo que vuelve aún menos virtuoso su desempeño en términos de sofisticación, al no tener mayores incentivos para procurar incrementar su rentabilidad vía incorporación de tecnología e innovaciones. 


\begin{tabular}{l|l|l|l}
\hline Rama & \multicolumn{2}{l}{$\mathbf{2 0 0 2}$} & \multicolumn{2}{l}{$\mathbf{2 0 0 8}$} & \multicolumn{2}{l}{$\mathbf{2 0 1 4}$} \\
\hline Alimentos y bebidas & $25,0 \%$ & $23,2 \%$ & $23,9 \%$ \\
Metalmecánica exc. Automotriz & $10,4 \%$ & $14,3 \%$ & $13,7 \%$ \\
Químicos & $12,7 \%$ & $11,3 \%$ & $12,2 \%$ \\
Metales & $9,8 \%$ & $9,2 \%$ & $8,6 \%$ \\
Confecciones & $5,6 \%$ & $5,8 \%$ & $5,7 \%$ \\
Automotriz & $3,6 \%$ & $5,0 \%$ & $4,9 \%$ \\
Refinación de petróleo & $7,5 \%$ & $4,9 \%$ & $4,5 \%$ \\
Caucho y plástico & $5,5 \%$ & $4,8 \%$ & $4,5 \%$ \\
Minerales no metálicos & $2,9 \%$ & $4,2 \%$ & $4,4 \%$ \\
Edición e impresión & $3,4 \%$ & $3,9 \%$ & $3,8 \%$ \\
Industrias manufactureras ncp & $2,4 \%$ & $2,8 \%$ & $3,2 \%$ \\
Textiles & $2,3 \%$ & $2,8 \%$ & $2,9 \%$ \\
Papel & $3,5 \%$ & $3,1 \%$ & $2,8 \%$ \\
Cuero y calzado & $2,2 \%$ & $2,3 \%$ & $2,6 \%$ \\
Madera & $2,5 \%$ & $2,0 \%$ & $2,1 \%$ \\
Tabaco & $0,8 \%$ & $0,5 \%$ & $0,5 \%$ \\
\hline PBI industrial & $100 \%$ & $100 \%$ & $100 \%$ \\
\hline
\end{tabular}

Cuadro $\mathbf{N}^{\circ}$ 2. Evolución de la participación de las distintas ramas industriales dentro del Producto Industrial (2002, 2008 y 2014)

Fuente: Elaboración propia sobre la base de Porta, Santarcángelo y Schteingart (2016).

\begin{tabular}{l|l|l|l|l}
\hline Año & \multicolumn{1}{l}{ Las 200 } & \multicolumn{1}{l}{ Primeras 50 } & \multicolumn{1}{l}{ Segundas 50 } & \multicolumn{1}{l}{ Segundas $\mathbf{1 0 0}$} \\
\hline 2003 & 26,9 & 17,4 & 5 & 4,6 \\
2004 & 26,7 & 16,7 & 5,1 & 4,8 \\
2005 & 26,5 & 16,8 & 4,9 & 4,8 \\
2006 & 26,6 & 16,9 & 5 & 4,7 \\
2007 & 26,4 & 16,6 & 4,9 & 4,8 \\
2008 & 25,9 & 16,4 & 4,8 & 4,7 \\
2009 & 24,6 & 15,4 & 4,6 & 4,6 \\
2010 & 23,6 & 14,9 & 4,4 & 4,3 \\
2011 & 23,8 & 14,7 & 4,6 & 4,5 \\
2012 & 23,5 & 14,5 & 4,6 & 4,4 \\
2013 & 24,9 & 15,9 & 4,6 & 4,5 \\
2014 & 23,2 & 14,9 & 4,1 & 4,2 \\
2015 & 22 & 13,8 & 4 & 4,2 \\
\hline
\end{tabular}

Cuadro $\mathbf{N}^{\circ}$ 3. Evolución de la participación de la cúpula empresarial local* en el valor bruto de producción total según tramos del ordenamiento, 20032015 (en porcentajes)

Fuente: Elaboración propia sobre la base de datos de Schorr (2018)

*Se trata de las 200 empresas más grandes del país de acuerdo con sus respectivas ventas anuales, no se incluyen firmas del sector financiero ni agropecuario, salvo las avocadas a la comercialización de granos. 


\begin{tabular}{l|l|l|l|l|l|l}
\hline & \multicolumn{4}{l|}{ Cantidad de empresas } & \multicolumn{3}{l}{ \% de ventas de la cúpula } \\
\cline { 2 - 7 } & 2001 & 2007 & 2015 & 2001 & 2007 & 2015 \\
\hline Estatal & 1 & 6 & 3 & 1,6 & 2,1 & 3,2 \\
Privada nacional & 59 & 53 & 63 & 25,3 & 22,8 & 27,6 \\
Privada extranjera & 93 & 116 & 114 & 55,2 & 64,1 & 51,4 \\
Asociación & 47 & 25 & 20 & 17,9 & 11 & 17,8 \\
\hline Total & 200 & 200 & 200 & 100 & 100 & 100 \\
\hline
\end{tabular}

Cuadro $\mathbf{N}^{\circ}$ 4. Distribución de las empresas y las ventas de la cúpula empresarial local según el tipo de accionista predominante de las firmas, 2001, 2007 y 2015 (en valores absolutos y porcentajes)

Fuente: Schorr (2018:136).

Vemos así, entonces, que el sistema productivo industrial se mantiene concentrado y transnacionalizado, y productivamente orientado hacia la producción de materias primas y manufacturas centradas en el procesamiento de recursos naturales y/o amparadas en ventajas institucionales de privilegio. No se ha podido avanzar en la consolidación de una estrategia de acumulación que, sobre la base del desenvolvimiento de un complejo industrial endógeno, complejo y dinámico, habilite formas de reproducción interna más equitativas, incluyentes y sostenibles, a partir de las cuales eludir la dependiente inserción al mercado global.

\section{Conclusiones}

Luego de examinar la configuración organizacional, la capacidad de fuego de la estructura estatal y la modalidad de las formas implicativas derivadas de la misma, se observa que el Estado argentino durante los años 2003-2015 no logró conformar una estatalidad coherente y cohesionada, ni desarrolló políticas públicas tendientes a conformar un sector industrial endógeno, complejo y dinámico, que tienda a propiciar el desarrollo.

El análisis de las transformaciones organizacionales, a pesar de la rejerarquización de las áreas vinculadas al fomento del sector industrial, dio cuenta que esas instancias ocupan un lugar marginal en el conjunto del organigrama del Estado Nacional. Ello se confirmó con el análisis de la clasificación institucional del gasto. En él se observó que las instancias ministeriales vinculadas al sector manufacturero presentaban una participación 
insignificante dentro del presupuesto de la Administración Nacional. A su vez, si bien según la clasificación por finalidad, la categoría servicios económicos presentaba un incremento a lo largo del período analizado, cuando se examinó la categoría funcional presupuestaria industria, se advirtió una muy baja gravitación de la misma.

El examen de la modalidad implicativa, por su parte, también dio cuenta de tendencias contradictorias. Por un lado, se observó la presencia de un conjunto de políticas de fomento al desarrollo, y específicamente a la actividad industrial y a sectores relevantes en ella, como las Pymes. Pero por otro lado, se advirtió que, a pesar que aumentaron en cantidad y en recursos, las mismas no conformaron una estrategia de intervención coherente y cohesionada, se trató más bien de un conjunto de acciones fragmentadas y desarticuladas entre sí.

En el artículo, además, se examinó la articulación entre la forma organizacional y la modalidad implicativa del Estado en Argentina durante los años 2003-2015 y la estrategia de acumulación. De acuerdo con ello, se advirtió que el aparato estatal viabilizó una estrategia de acumulación orientada hacia la producción de manufacturas amparadas en las ventajas comparativas de los recursos naturales y/o en ventajas institucionales de privilegio, realizadas por un reducido número de empresas crecientemente propiedad de capitales extranjeros, lo que acarrea limitadas posibilidades de generar dinámicas de crecimiento con inclusión social.

En función de lo expuesto se destaca, como una de las limitaciones de la reacción neodesarrollista argentina la no construcción de una estatalidad que desde su forma organizativa y lógica implicativa viabilice procesos de desarrollo. Por lo tanto, se resalta la importancia teórica, académica y política de la estructura estatal y las políticas públicas derivadas de ella en la configuración de una estrategia de acumulación que habilite la salida de posicionamientos periféricos. 


\section{Referencias bibliográficas}

AMSDEN, A. (1989) Asia's next giant: South Korea and late insdustrialization, Oxford: Oxford University Press.

- (2004). La sustitución de importaciones en las industrias de alta tecnología: Prebisch renace en Asia. Revista de Ia CEPAL, 82(Abril), 75-90.

ARANÍBAR, A. \& RODRÍGUEZ, B. (2013). América Latina ¿del neoliberalismo al neodesarrollismo? Buenos Aires: Siglo XXI.

ARCEO, E. (2005). El impacto de la globalización en la periferia y las nuevas y viejas formas de dependencia en América Latina. Cuadernos del CENDES, 22, 27-63.

ARRIGHI, G., SILVER, B. Y BREWER, B. (2003). Industrial Convergence, Globalization, and the Persistence of North-South Divide. Studies in Comparative International Development, 3-31.

ÁVILA GOMIDE, A. Y BOSCHI, R. (ed.) (2016) Capacidades estataisem países emergentes: o Brasil em perspectiva comparada. Rio de Janeiro: IPEA.

AZPIAZU, D. Y SCHORR, M. (2010) Hecho en Argentina. Industria y economía, 19762007, Buenos Aires: Siglo XXI Editores.

BARUJ, G. Y PORTA, F. (2006). Políticas de competitividad en la Argentina y su impacto sobre la profundización del Mercosur. Documento de Proyecto. Santiago de Chile: CEPAL.

BARUJ, G., KOSACOFF, B. Y RAMOS, A. (2009). Las políticas de promoción de la competitividad en la Argentina. Principales instituciones e instrumentos de apoyo y mecanismos de articulación público-pri- vada, Documento de Proyecto, Santiago de Chile: CEPAL.

BRESSER-PEREIRA, L.C. (2006). EI nuevo desarrollismo y la ortodoxia convencional, Economía vol. 4, n 10. México DF, Universidad Autónoma de México.

(2007). Estado y mercado en el nuevo desarrollismo, Nueva Sociedad 210. Caracas.

CAO, H., REY, M. Y LAGUADO LUCA, A. (2015). El Estado en cuestión. Ideas y política en la Administración Pública Argentina 19582015. Buenos Aires: Prometeo.

CASTELLANI, A. Y LLAMPART, F. (2012.) Debates en torno a la calidad de la intervención estatal, Papeles de Trabajo 6, n 9 , junio.Revista electrónica del Instituto de Altos Estudios Sociales de la Universidad Nacional de General San Martín, Buenos Aires.

CASTELLS, M. Y SCHORR, M. (2015). Cuando el crecimiento no es desarrollo. Algunos hechos estilizados de la dinámica industrial en la posconvertibilidad, Cuadernos de Economía Crítica 2. Buenos Aires.

CEPAL (1951). Estudio económico de América Latina. Nueva York: Naciones Unidas.

CHAN, H. (2004). Retirar la escalera. La estrategia de desarrollo en perspectiva histórica. Madrid: Catarata.

CHIBBER, V. (2002). Bureaucratic Rationality and the Developmental State, American Journal of Sociology, Vol 107, $n^{\circ} 4$. COHEN, S. Y ZYSMAN, J. (1987). Manufacturing Matters. The myth of the post-industrial economy. New York: Basic Books. 
COUTO, B. (2010). Desafíos para el Estado y los actores empresarios en la definición e implementación de la política industrial en Argentina, Documentos de Investigación Social 11, IDAES, UNSAM.

ESPAÑOL, P. Y HERRERA, G. (2011). La (re) construcción de un proyecto nacional para el desarrollo. Algunas notas para alentar la discusión, Revista de Ciencias Sociales 3, n 19, Bernal, Universidad Nacional de Quilmes, Segunda Época.

EVANS, P. (1995). Embedded Autonomy. States \& Industrial Transformation. Princeton: Princeton University Press.

- (1996). El Estado como problema y solución en Desarrollo Económico Revista de Ciencias Sociales 35, n 140, Buenos Aries: IDES.

FAJNZYLBER, F. (1983). La industrialización trunca de América Latina. México: Nueva Imagen.

(1992). Industrialización en América Latina. De la caja negra al casillero vacío. Nueva Sociedad, 118, 21-28.

FERNÁNDEZ BUGNA, C. Y PORTA, F. (2007). EI crecimiento reciente de la industria argentina. Nuevo régimen sin cambio estructural. En Kosacoff, Bernando (comp.) Crisis, recuperación y nuevos dilemas. La economía argentina 2002-2007, Santiago de Chile: CEPAL.

FERNÁNDEZ, V.R. (2017). La trilogía del erizozorro. Redes globales, trayectorias nacionales y dinámicas regionales desde la periferia. Barcelona: Anthropos-Siglo XXI Editores-Ediciones UNL.
FERNÁNDEZ, V.R. Y LAUXMANN, C. (2014). ¿Cuál(es) camino(s) conduce(n) a Roma? Estado y políticas industriales en los desafíos del desarrollo latinoamericano. Cuadernos del CENDES, 86, 49-72.

FERNÁNDEZ, V.R., GÜEMES, M.C., MAGNIN, J. Y VIGIL, J. (2006) Capacidades estatales y desarrollo regional. Realidades $y$ desafíos para América Latina, Santa Fe: Ediciones UNL.

FERRER, A. (2010). Raúl Prebisch y el dilema del desarrollo en el mundo global. Revista de la CEPAL 101, agosto.

GAITÁN, F. (2013). El rescate del Estado. Los desafíos del desarrollo, Buenos Aires: Capital Intelectual.

- (2014) Auge, ocaso y resurgimiento de Ios estudios sobre desarrollo en América Latina. Santiago de Chile: CEPAL.

GARCÍA PUENTE, M.J. (2016) Transformaciones del Estado y desarrollo. Capacidades estatales y desarrollo productivo industrial. El caso de la provincia de Santa Fe en el período 1990-2013. Tesis de doctorado. Universidad Nacional de Rosario. GREENWALD, B. Y STIGLITZ, J. (2006). Helping Infant Economies Grow: Foundations of Trade Policies for Developing Countries. New Developments in Macroeconomics 96, 141-146.

HAUSMANN, R. Y RODRIK, D. (2003). Economic development as self-discovery. Journal of Development Economics, 72, 603-633.

HELPER, S., KRUEGER, T. Y WIAL, H. (2012). Why Does Manufacturing Matters? Which 
Manufacturing Matters? A Policy Framework. In Metropolitan Policy Program at Brookings.

HIKINO, T. Y AMSDEN, A. (1995). La industrialización tardía en perspectiva histórica, Desarrollo Económico, 35, 3-34.

KOLHI, A. (2010). Estado y desarrollo económico, Documentos y Aportes en Administración Pública y Gestión Estatal $n^{\circ}$ 12. Universidad Nacional del Litoral. Santa Fe.

KULFAS, M. (2009). Las Pymes argentinas en el escenario post convertibilidad. Políticas públicas, situación y perspectivas, Colección Documentos de Proyectos. LC/W.272, Santiago de Chile: CEPAL. (2016). Los tres kircherismos. Una historia de la economía argentina 20032015. Buenos Aires: Siglo XXI.

LAUXMANN, C.T. Y FERNÁNDEZ, V.R. (2015). Industrialización y desarrollo en la Argentina pos-"Washington Consensus": un abordaje crítico desde la perspectiva sectorial, actoral y espacial. Semestre Económico, 18(37), 51-70.

LAVARELLO, P. (2017). ¿De qué hablamos cuando hablamos de política industrial? En M. Abeles, M. Cimoli, y P. Lavarello (Eds.), Manufactura y cambio estructural. Aportes para pensar la política industrial en la Argentina. Santiago de Chile: CEPAL.

LAVARELLO, P. Y GOLDSTEIN, E. (2011). Dinámicas heterogéneas en la industria de maquinaria agrícola argentina, Problemas del Desarrollo. Revista Latinoamericana de Economía 42, núm. 166. Distrito Federal, México, Universidad Nacional Autónoma de México.
MANN, M. (1991). El poder autónomo del Estado: sus orígenes, mecanismos y resultados, Zona Abierta $n^{\circ} 57 / 58$.

MINISTERIO DE INDUSTRIA (2011). Plan Estratégico Industrial 2020. Buenos Aires: Presidencia de la Nación.

NAHÓN, C., RODRÍGUEZ, E. Y SCHORR, M. (2006). El pensamiento latinoamericano en el campo del desarrollo del subdesarrollo: trayectorias, rupturas y continuidades. Crítica y Teoría en Pensamiento Social Latinoamericano. CLACSO.

PINTO, A. (1973). Heterogeneidad estructural y modelo de desarrollo reciente de la América Latina. ILPES - CEPAL.

PORTA, F., SANTARCÁNGELO, J. Y SCHTEINGART, D. (2016) Producción y empleo en el sector industrial argentino: 18892014. H-industri@ 10(19), segundo semestre.

PREBISCH, R. (1949). El desarrollo económico de la América Latina y algunos de sus principales problemas. Desarrollo Económico 26(103).

ROUGIER, M. Y SCHORR, M. (2012). La industria en los cuatro peronismos. Estrategias, políticas y resultados, Buenos Aires: Capital Intelectual.

SCHORR, M. (2013) (coord.) Argentina en a posconvertibilidad: ¿desarrollo o crecimiento industrial? Estudios de economía política. Buenos Aires: Miño y Dávila Editores.

- (2018). El poder económico de la Argentina bajo los gobiernos kirchneristas. Un análisis a partir del panel de grandes empresas. En M. Schorr (Coord.) Entre la década ganada y la década per- 
dida. La argentina kirchnerista ensayos de política económica. Buenos Aires: Batalla de ideas.

SEVARES, J. (2010). ¿Por qué crecieron los países que crecieron? Historias y teorías del crecimiento económico. Realidad Económica 253, 20-47.

SIKKINK, K. (1993). Las capacidades y la autonomía del Estado en Brasil y la Argentina. Un enfoque institucionalista, Desarrollo Económico. Revista de Ciencias Sociales 32, $n^{\circ} 128$. Buenos Aires.

SKOCPOL, T. (1991). El Estado regresa al primer plano: Estrategias de análisis en la investigación actual, Zona Abierta 57/58.

WADE, R. (1999) El mercado dirigido. La teoría económica y la función del gobierno en la industrialización del este de Asia. México: Fondo de Cultura Económica. 


\section{Jimena García Puente Doctora en Ciencia Política (Universidad Nacional de Rosario) Magíster en Adminis- tración y Políticas Públicas (Universidad de San Andrés) Licenciada en Ciencia Política (UNR). Docente - Investigadora de la Univer- sidad Nacional del Litoral y docente de la Universidad Nacional de Entre Ríos.}

\section{Carolina T. Lauxmann}

Doctoranda en Ciencias Sociales (Universidad de Buenos Aires). Magíster en Histórica Económica y Política Económica (FCE-UBA). Docente-Investigadora de la Facultad de Ciencias Económicas y Humanidades y Ciencias de la Universidad Nacional del Litoral.

\section{Emilia Ormaechea}

Doctoranda en Desarrollo Económico (Universidad Nacional de Quilmes). Becaria del CONICET. Magíster en Ciencias Sociales y Licenciada en Ciencia Política (Universidad Nacional del Litoral). Docente de la Facultad de Ciencias Jurídicas y Sociales, y de la Facultad de Humanidades y Ciencias de la Universidad Nacional del Litoral.

\section{REGISTRO BIBLIOGRÁFICO}

María Jimena García Puente, Carolina Teresita Lauxmann y Emilia Ormaechea "EL ESTADO ARGENTINO Y EL DESARROLLO. UN ANÁLISIS DE LA CONFIGURACIÓN E IMPLICACIÓN ESTATAL EN LA PROMOCIÓN INDUSTRIAL DURANTE LOS AÑOS 2003-2015", en Papeles del Centro de Investigaciones, Facultad de Ciencias Jurídicas y Sociales, UNL, publicación semestral, año 9, número 20, Santa Fe, República Argentina, 2019, pp. 9-30. 